\title{
Computational system to create an entry file for replicating I-125 seeds simulating brachytherapy case studies using the MCNPX code
}

\author{
Leonardo S. Boia' ${ }^{1}$, Juraci P. R. Junior' ${ }^{1}$, Artur F. Menezes ${ }^{1}$, Ademir X. Silva ${ }^{1,2}$ \\ ${ }^{I}$ Programa de Engenharia Nuclear/COPPE, Av. Horácio Macedo, Universitária, Ilha do Fundão, Rio de Janeiro, Brasil. \\ ${ }^{2}$ Departamento de Engenharia Nuclear/Escola Politécnica, Av. Horácio Macedo, Universitária, Ilha do Fundão, RJ, Brasil.
}

Received January 24, 2014; Revised February 25, 2014; Accepted March 11, 2014; Published Online March 25, 2014

\section{Original Article}

\begin{abstract}
Purpose: A computational system was developed for this paper in the $\mathrm{C}++$ programming language, to create a ${ }^{125} \mathrm{I}$ radioactive seed entry file, based on the positioning of a virtual grid (template) in voxel geometries, with the purpose of performing prostate cancer treatment simulations using the MCNPX code. Method: The system is fed with information from the planning system with regard to each seed's location and its depth, and an entry file is automatically created with all the cards (instructions) for each seed regarding their cell blocks and surfaces spread out spatially in the 3D environment. The system provides with precision a reproduction of the clinical scenario for the MCNPX code's simulation environment, thereby allowing the technique's in-depth study. Results and Conclusion: The preliminary results from this study showed that the lateral pe- numbra of uniform scanning proton beams was less sensitive In order to validate the computational system, an entry file was created with $88{ }^{125}$ I seeds that were inserted in the phantom's MAX06 prostate region with initial activity determined for the seeds at the $0.27 \mathrm{mCi}$ value. Isodose curves were obtained in all the prostate slices in $5 \mathrm{~mm}$ steps in the 7 to $10 \mathrm{~cm}$ interval, totaling 7 slices. Variance reduction techniques were applied in order to optimize computational time and the reduction of uncertainties such as photon and electron energy interruptions in $4 \mathrm{keV}$ and forced collisions regarding cells of interest. Through the acquisition of isodose curves, the results obtained show that hot spots have values above $300 \mathrm{~Gy}$, as anticipated in literature, stressing the importance of the sources' correct positioning, in which the computational system developed provides, in order not to release excessive doses in adjacent risk organs. The $144 \mathrm{~Gy}$ prescription curve showed in the validation process that it covers perfectly a large percentage of the volume, at the same time that it demonstrates a large decline for short distances.
\end{abstract}

Keywords: C++ programming language; Brachytherapy; Phantom MAX06; Voxel; MCNPX

\section{Introduction}

According to estimates provided by Brazil's National Cancer Institute (INCA/RJ/Brazil) on the incidence of cancer in Brazil for the year 2012, 60,180 new cases of prostate cancer are expected. These values correspond to an estimated risk of 62 new cases per 100,000 men. ${ }^{1}$ The latest global estimate iden-

\footnotetext{
Corresponding author:

Leonardo S. Boia; Programa de Engenharia Nuclear/COPPE, Av. Horácio Macedo, 2030, Bloco G - Sala 206 - Centro de Tecnologia Cidade, Universitária, Ilha do Fundão, 21941-914 - Rio de Janeiro, RJ, Brasil. Email: lboia@con.ufrj.br

Cite this article as:

Boia LS, Junior J, Menezes AF, Silva AX. Computational system to create an entry file for replicating I-125 seeds simulating brachytherapy case studies using the MCNPX code. Int JCancer Ther Oncol2014; 2(2):02023. DOI: 10.14319/ijcto.0202.3
}

tifies prostate cancer as the second most common type of cancer in men, with roughly 915,000 new cases in 2008. Approximately $75 \%$ of all diagnosed cases occur in developed countries. The prostate cancer rate worldwide has increased about 25 times, with the highest rates observed in Australia, New Zealand, Western Europe and North America. ${ }^{1}$

In Brazil, the increase in life expectancy, the improvement and evolution of diagnostic methods, and the quality of information systems may explain the increase in incidence rates over the years. ${ }^{1}$ For this reason, the study and development of computational systems in the area of healthcare has shown considerable growth, vis-à-vis the need to improve the quality of treatments, which are carried out based on information and computational data obtained from 2D and 3D imaging. 
Due to the possibility of performing complex computational modeling in realistic virtual environments, computer simulations using the Monte Carlo method ${ }^{2}$ have been widely used in the area of medical physics aimed at providing tools that can be used to generate auxiliary data, the purpose of which is to assist the medical staff in seeking the best parameters for radiotherapy treatments. Obtaining reliable results during computational simulations is directly linked to the reproduction of a clinical scenario in a virtual environment, considering the geometry's correct modeling as well as the chemical composition's definition and the density of the materials employed.
A system was developed and assessed during this exercise named IS - Seed Insertion - in the $\mathrm{C}++$ programming language ${ }^{3}$, which reproduces the experimental template (Figure 1) employed for the ${ }^{125} \mathrm{I}$ seeds' three-dimensional insertion during the prostate cancer treatment, in order to provide a tool allowing the automation and reproduction of the clinical scenario during low-dosage rate (LDR) brachytherapy procedures in a computational environment employing the Monte Carlo MCNPX code.

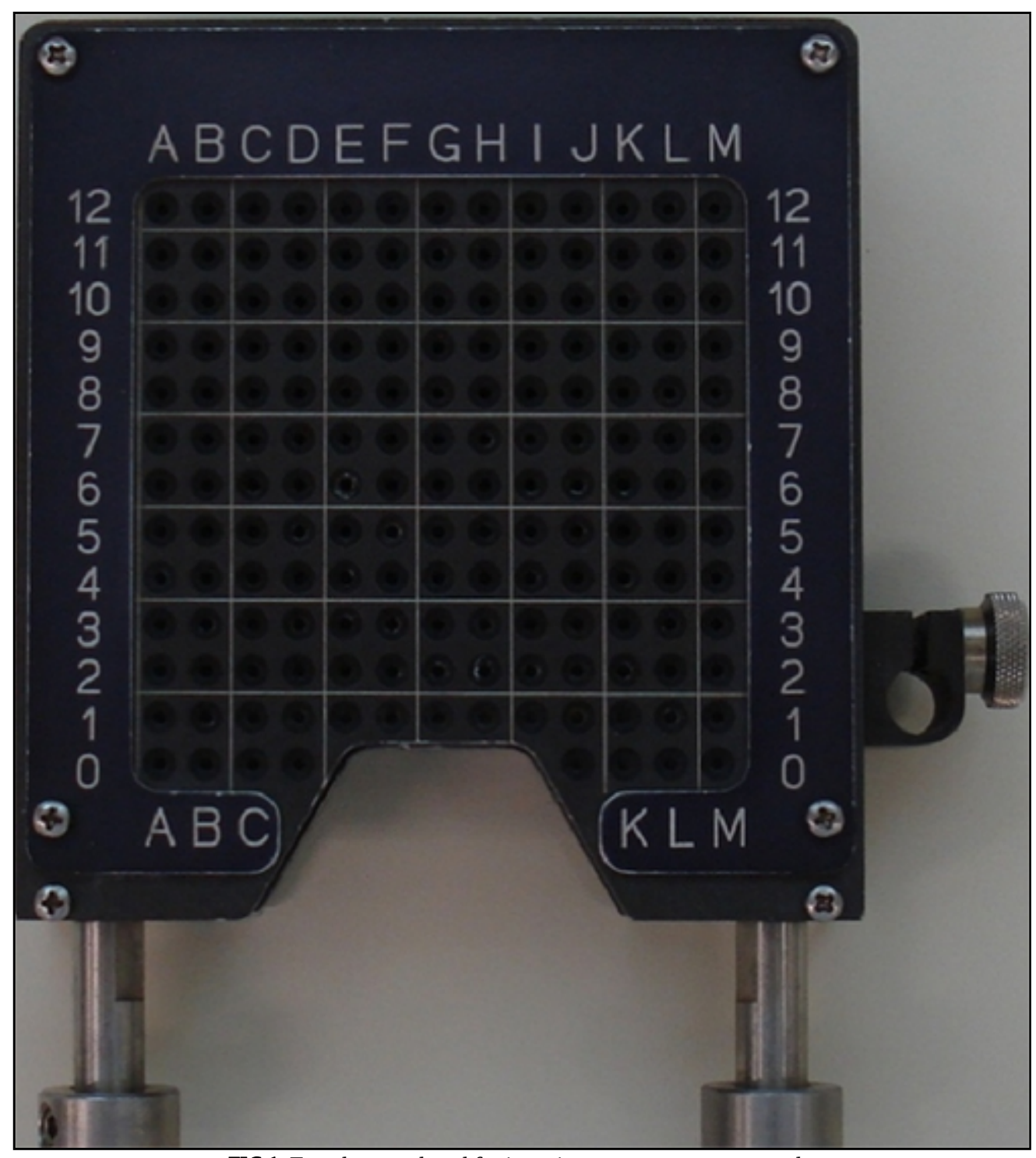

FIG.1: Template employed for inserting prostate treatment seeds. 
The IS program's use (Figure 2) developed in the C++ language jointly with the MCNPX simulation code allows performing more realistic computational simulations bearing in mind the seeds' correct three-dimensional positioning, in the variation of case studies due to a computational environment's freedom, and also allows a drastic reduction of the time spent to replicate a large number of seeds using the MCNPX code.

\section{Materials for Analysis}

\section{I Seeds}

Brachytherapy consists in a barely invasive procedure, by means of which radioactive seeds are implanted permanently in the patient under treatment. The ${ }^{125}$ I source (Figure 3 ) has been broadly employed in different radiotherapy clinics during permanent implants in order to treat some types of tumors, in particular prostate cancer. ${ }^{4}$

Differently from teletherapy, in which a source is located outside the patient, in brachytherapy the source is positioned inside or close to the region under treatment. Owing to the likelihood of obtaining the dose's high decreasing gradient, brachytherapy allows administering a localized high radia- tion dose, thus reducing the dose on adjacent side organs. ${ }^{4}$ Brachytherapy is employed to treat cancer in different regions of the body, among which may be mentioned prostate, cervix and eyes, among others. ${ }^{4}$

Please note that inserting ${ }^{125}$ I seeds is a clinical and randomized process, and it is not possible to predict whether the inserted source will be placed exactly in the position planned or whether a rotation will occur with it. Materials such as Vycril are placed in the source to prevent it from rotating in any of the three directions. The responsibility for the sources' exact positioning is the physician's, and the positioning's reproduction is the doctor's responsibility, who albeit assisted by the ultrasound image, minor uncertainties may occur during surgery.

The Amersham model $6711^{125}$ I source employed as a reference in this paper was modeled as consisting in an internal silver cylinder containing an emitting film $1 \mu_{\mathrm{m}}$ in thickness, with an outer casing in cylindrical form made of titanium $0.81 \mathrm{~mm}$ in diameter and a length of $4.5 \mathrm{~mm}$, closed by two ellipsoids. ${ }^{5}$

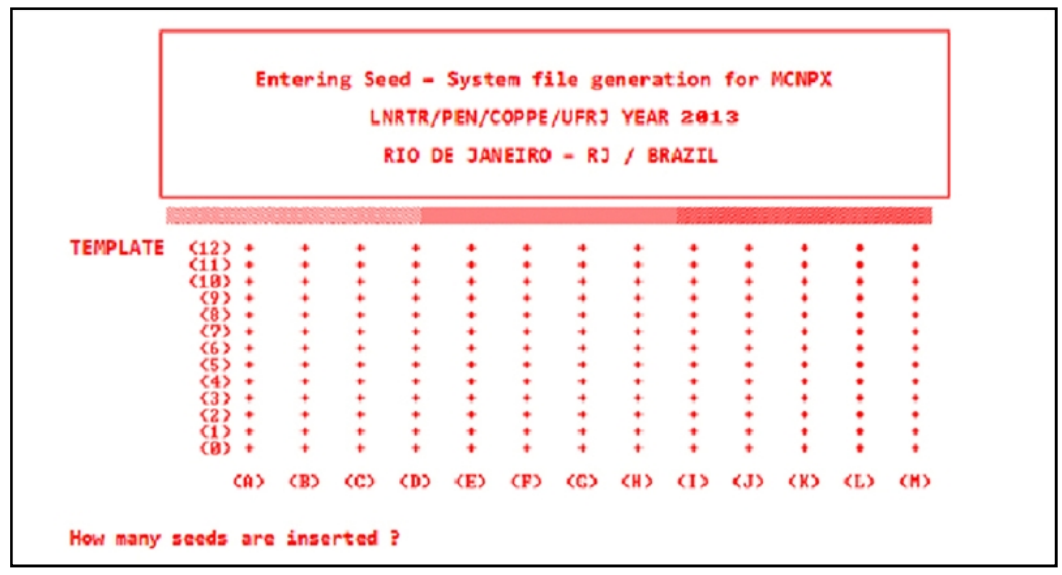

FIG.2: IS (Seed Insertion) computational system.

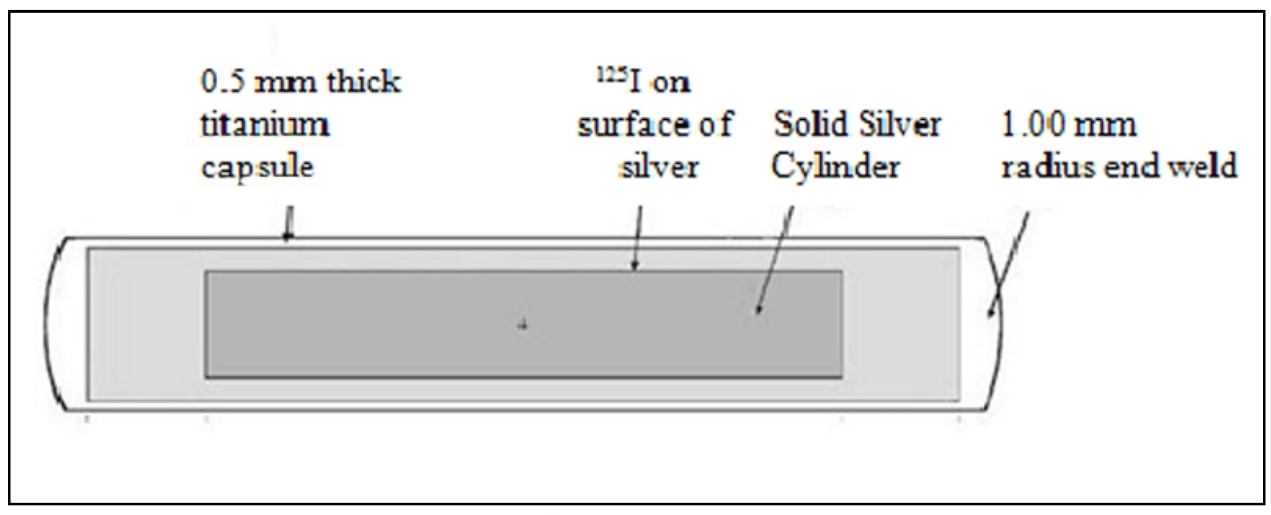

FIG. 3: The ${ }^{125}$ I seed's internal structure. 


\section{The MAX06 phantom}

The MAX (Male Adult Voxels) phantom was develop by Kramer et al. ${ }^{6}$, and is based on segmented images of a male adult provided by Zubal et al.7. The MAX06 phantom is an update of the original MAX phantom, where structures such as bronchi, lymphonodes and prostate were included. There was also a modification in the voxel size from $3.6 \mathrm{~mm}$ to 1.2 $\mathrm{mm} .^{8}$ This reduction led to an increase in the number of voxels by a factor of 27 , which can preclude computational simulations even for some high performance computers. Therefore, in order to surpass computational limitations and save time, in this work the software Tomo_MC ${ }^{9}$ was used to select some phantom slices of interest to the study: 154 along the $x$-axis $(18.48 \mathrm{~cm}) ; 163$ along the $y$-axis $(19.56 \mathrm{~cm})$ and 131 slices along the $z$-axis $(15.72 \mathrm{~cm})$. This selection resulted in 3.288.362 voxels instead of 150 million, the original number of MAX06 voxels (Figure 4).

\section{Computational process}

\section{Computational system's development}

The construction process for the cells' cards and ${ }^{125}$ I seeds' surfaces in an MCNPX code entry file is extremely elaborate when created manually. If for example the purpose was to create 100 seeds in an MCNPX code entry file, innumerous lines of manually digitized instructions would be required with their respective calculations to be handled individually, which could possibly give rise to errors and adversely affect the simulation's final results.

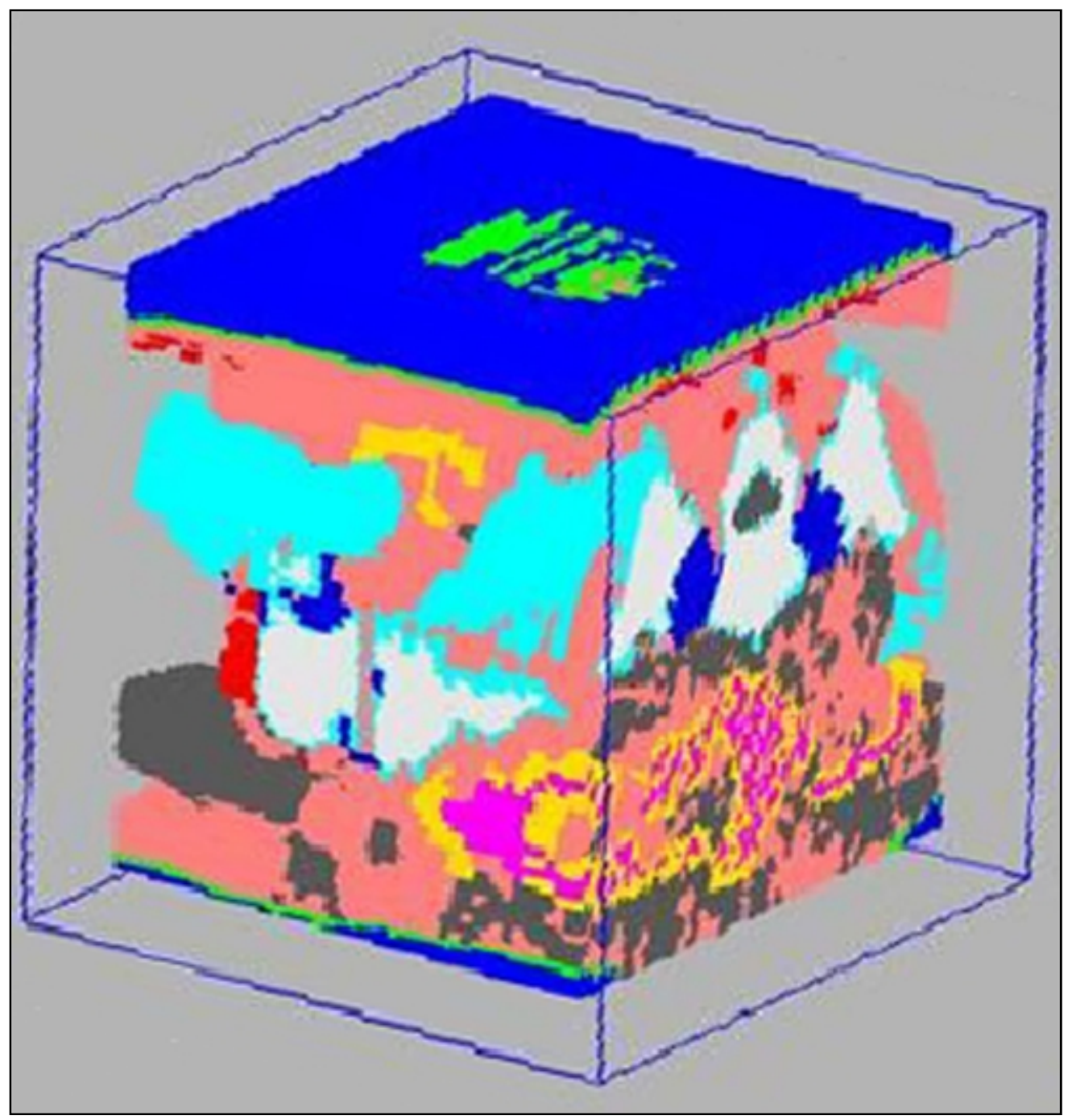

FIG. 4: The phantom MAX06's X, Y and Z cuts made by the TOMO_MC program. 


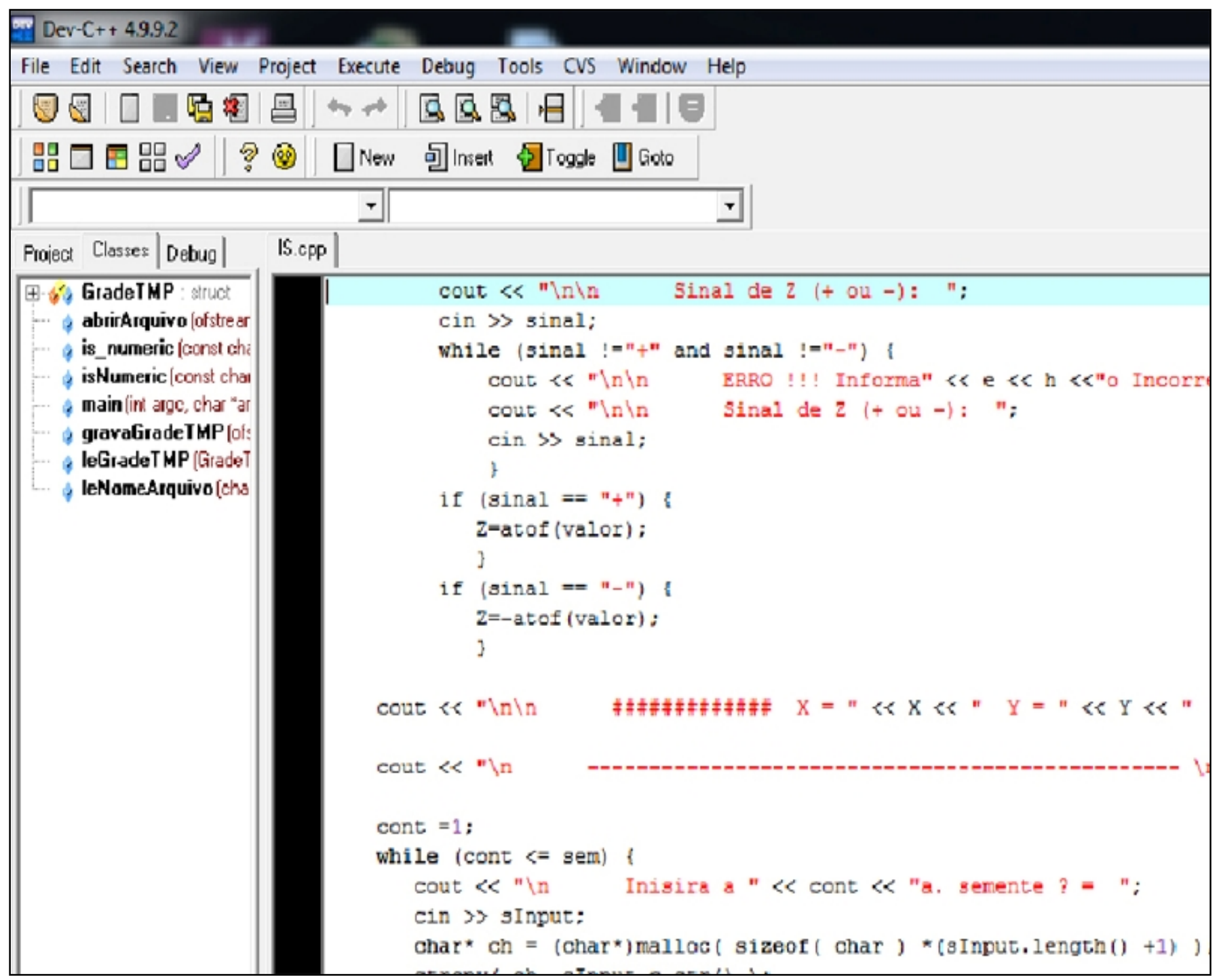

FIG. 5: Dev-C++ development environment.

In the doctoral thesis "Assessments of the approximations employed in planning prostate brachytherapy treatment" by Reis ${ }^{4}$, a 7- month period was required to build an entry file of $88^{125}$ I seeds, in addition the likelihood of error in preparing the entry file is large when compared to the program, which shows the complexity of the entry file's building process. In order to create an entry file, with the construction of cells and surfaces and of its data calculated and automatically positioned based on data on the seeds fed into the system, a program was prepared in the $\mathrm{C}++$ programming language employing a free integrated $\mathrm{Dev}-\mathrm{C}++{ }^{10}$ (Figure 5) development environment that employs GNU project compilers to compile programs for the Microsoft Windows operating system.
The program's code employs multi-dimensional variables that allow storage of a set of data of the same type in different dimensions. Ordering and sequencing the control structures determine the format of actions such as for example: field protection, performance of assembly calculations for MCNPX code instruction cards and saving data in entry files. The fstream (file stream) library present in the program code allows handling data in files and creating objects that will serve as intermediaries between the program and the files handled. The basic flowchart of the algorithm IS system is shown in Figure 6. 


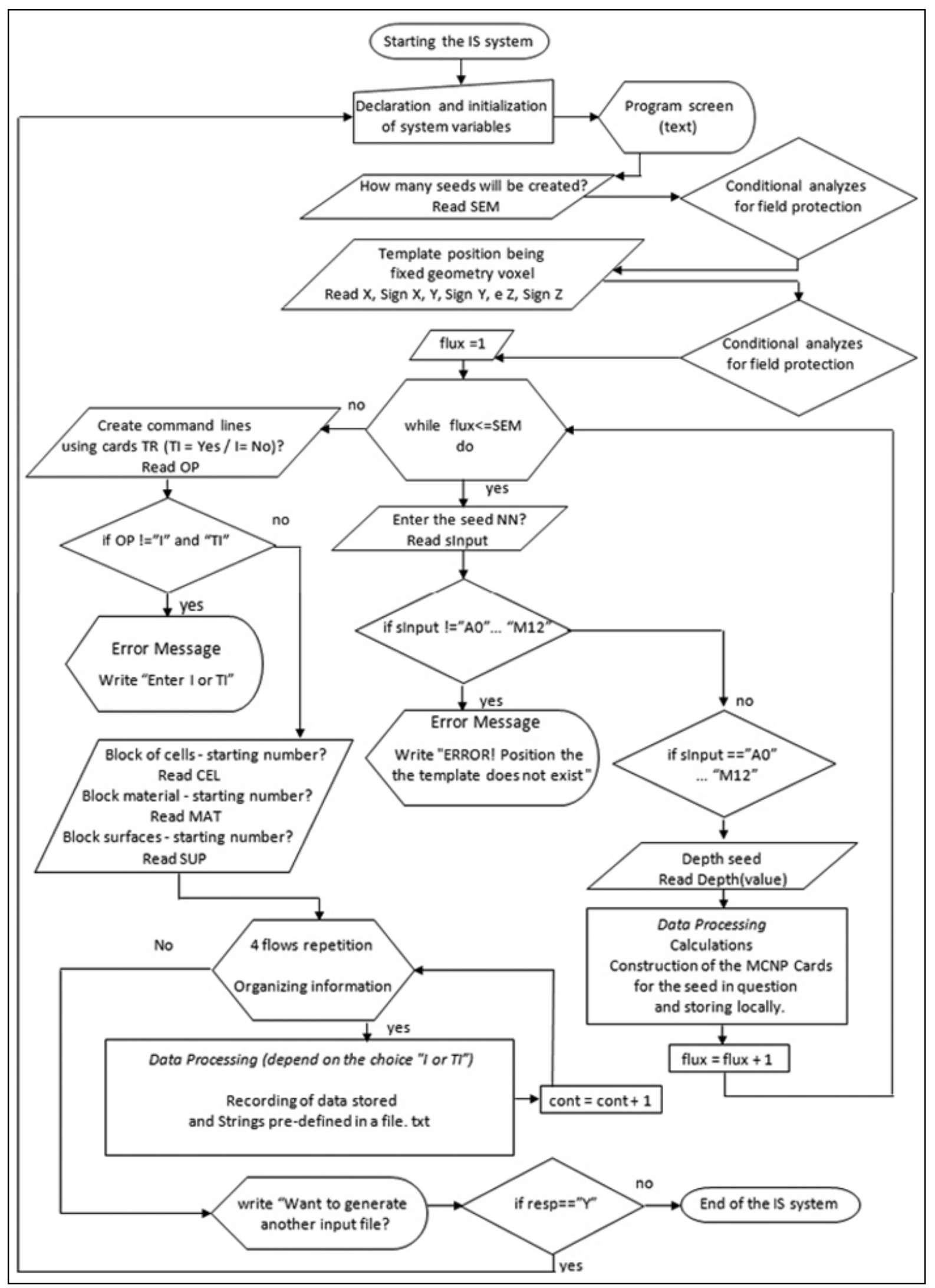

FIG. 6: Basic flowchart of the IS system. 


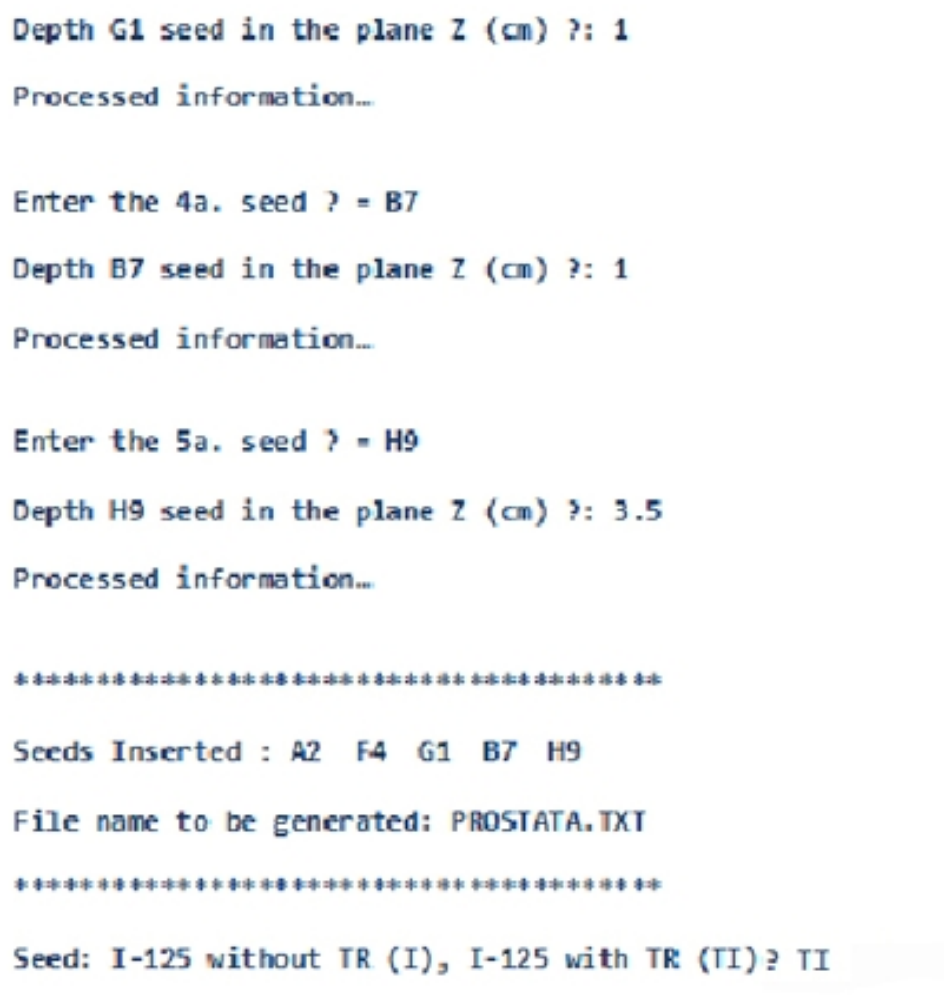

FIG. 7: Seed insertion system display requesting user to inform the instruction format to be created in the entry file (with or without use of the TR card).

When running the file, the data positioned in the reference planning are informed according to the requests submitted by the IS system. The initial question made by the program to the operator is on the amount of seeds to be employed in the treatment and where will be the grid's initial location so that it may be positioned pursuant to the lattice structure's spatial distribution. This position's precise information is obtained through the Moritz Geometry Tool ${ }^{11}$, program, which allows visualizing in $3 \mathrm{D}$ the voxel structure's geometry. This process being performed, the system requests the first seed's position, which is informed by the reference planning. Columns in the template are represented by the letters $\mathrm{A}$ to $\mathrm{M}$ and lines are represented by numerals from 0 to 12 . If it is informed to the system that the first seed is located in "F3", it will automatically position the cards (instructions) of the respective seed, and then it will request this seed's depth in the prostate or in the desired organ. The seed's depth in the coordinate $\mathrm{Z}$ is informed by the user, and the IS system will automatically determine the instructions (orders) for its translation, and in this same manner the process will proceed in the same manner for the following seeds.

After inserting the seeds in the desired positions and depths, the system will request to be informed of the name of the
.TXT format file to be created and the instruction format to be created for the MCNPX entry file. Format options are: I-125 without TR and I-125 with TR. (Figure 7).

Embedded in the IS system, using the transformation card (TR Card) found in the MCNPX code, allows positioning or rotating the ${ }^{125} \mathrm{I}$ modeled seeds, providing a dynamism in replicating the seeds, the positions and amounts of which are previously defined, using as a reference seeds defined in the source of the coordinates system. Based on the link established between the surfaces of the seeds defined at the source and the transformation card (TR Card) employed, the IS system will position the seeds to the respective previously planned positions.

After all the seeds are inserted, the cell block's initial number is requested, as well as the materials' initial number and the surface block's initial number. Once these latter fields are informed, the entry field is created as shown in Figure 8, and to finalize the program, the user is asked if it wishes to continue creating another entry file or to exit, thus finalizing the computational process. 


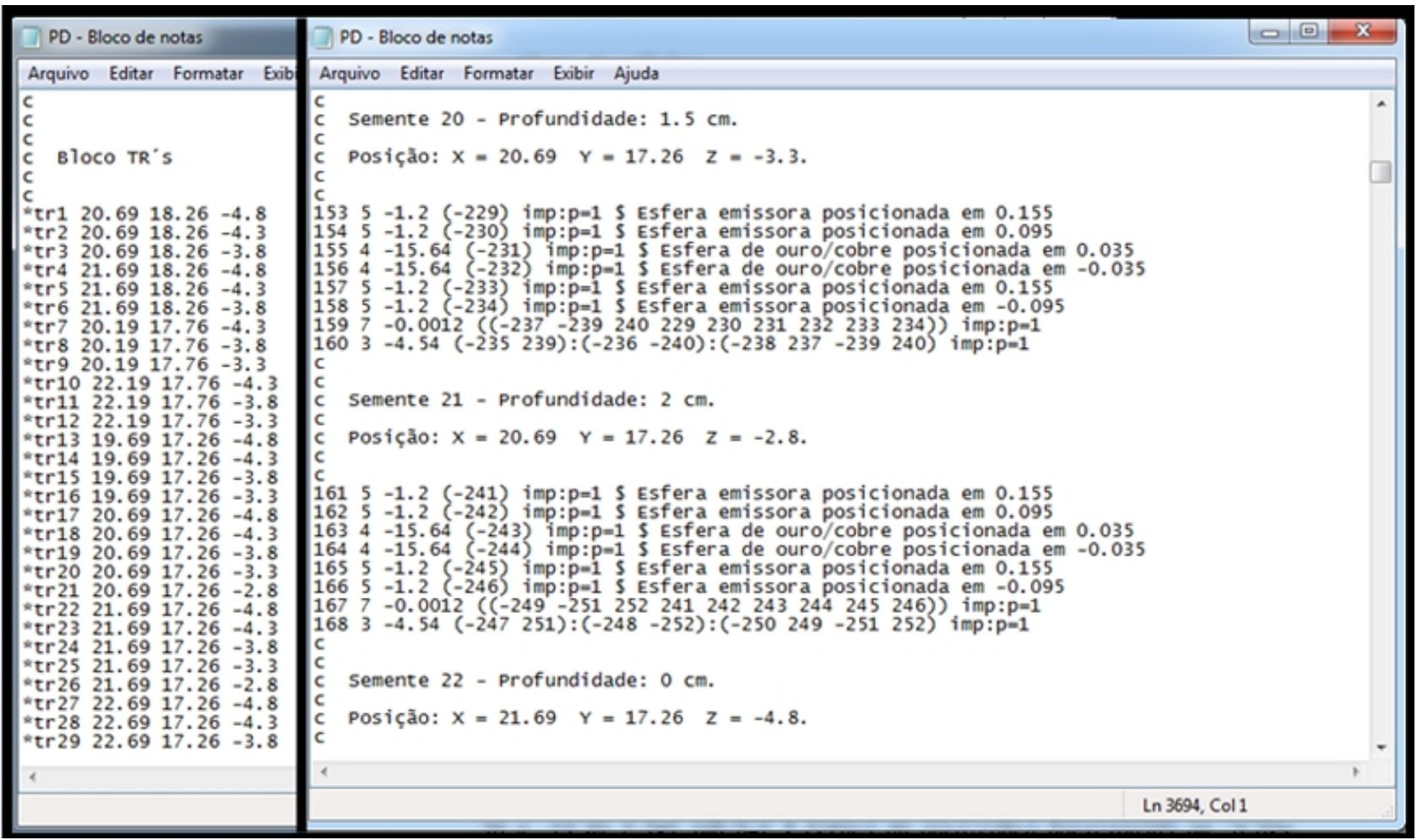

FIG. 8: Parts of the entry file created by the IS system for the MCNPX code.

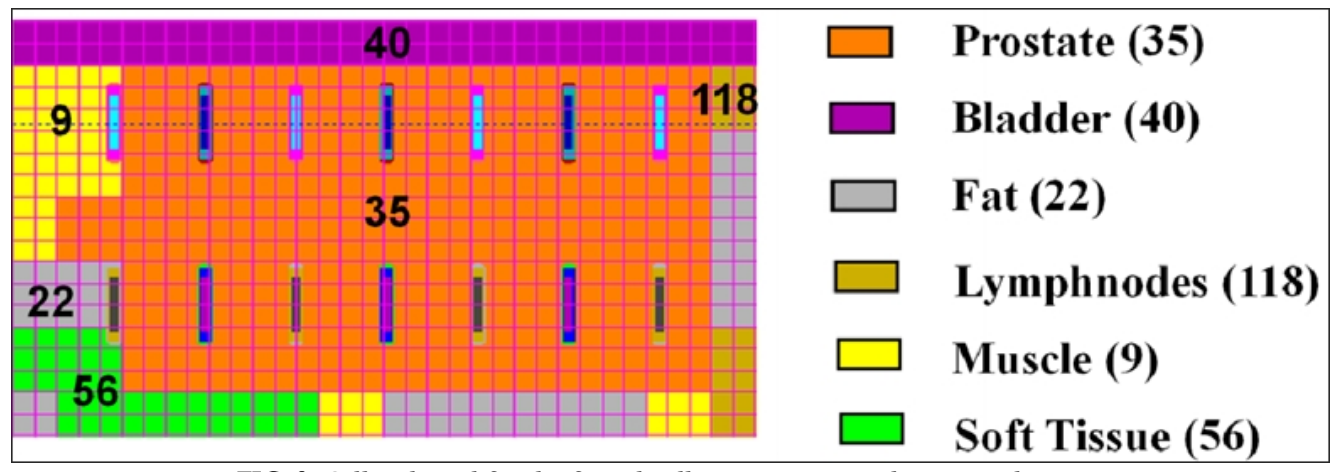

FIG. 9: Cells selected for the forced collision variance reduction technique.

\section{IS system validation}

In order to validate the IS system, the system was fed with literature data ${ }^{12}$ through the insertion of $88{ }^{125}$ I seeds (the average number of seeds estimated for a prostate implant), and an entry file is created with all the seeds positioned in the phantom MAX06's prostate region, with initial activity determined for the seeds at the $0.27 \mathrm{mCi}$ value. Isodose curves were obtained in all the prostate slices in $5 \mathrm{~mm}$ steps in the 7 to $10 \mathrm{~cm}$ interval, totaling 7 slices. The number of histories applied to the simulation was $200,000,000$, totaling a 2 -day value.

Variance reduction techniques were applied in order to optimize computational time and the reduction of uncertainties such as photon and electron energy interruptions (cut:p and cut:e) in $4 \mathrm{keV}$ and forced collisions regarding prostate cells and adjacent tissues with their respective identifications (IDs) as shown in Figure 9.

Figure 8 illustrates the values found for the total dose delivered to the prostate implant when Monte Carlo simulations are used to mock up real geometry and chemical composition of the structures for the configuration containing 88 seeds with radiation activity of $0.27 \mathrm{mCi}$, an absorbed dose of 146 Gy was found, very close to the AAPM recommendation ${ }^{13}$. The number of transformations for 88 seeds of I-125 with activities of $0.27 \mathrm{mCi}$. Initial activity Number of transformations (dis) 88 seeds $6.51 \times 10^{15}$ dis. 
The deposition of total radiation in permanent prostate implants is proportional to the radionuclide's disintegration, and the total number of transformations is given by the equation $(1)$, with an integer in the time interval $(t=0)$ the $(t=\infty)$ :

$$
\int_{0}^{\infty} A_{0} \cdot e^{-\lambda . t} \Longrightarrow-\frac{A_{0}}{\lambda} \cdot\left(\frac{1}{e^{\lambda . \infty}}-\frac{1}{e^{\lambda .0}}\right) \Longrightarrow \frac{A_{0}}{\lambda} .
$$

\section{Isodose curves for a selected MAX06 slices}

From the analysis of the results presented in Figure 8, it was concluded that the configuration that delivers absorbed doses closest to the recommended value is the set of 88 seeds with initial activity of $0.27 \mathrm{mCi}$. Therefore, for the construction of isodose curves, each voxel was normalized using this configuration as reference. Figure 10 below reflects the isodose curves for each slice of the phantom MAX06's prostate region and the respective values / colors shown in the sub-title. The isodose curves were placed in multiples of 36 , as the idea is to show that the 144 Gy curve covers the larger portion of the prostate's volume.

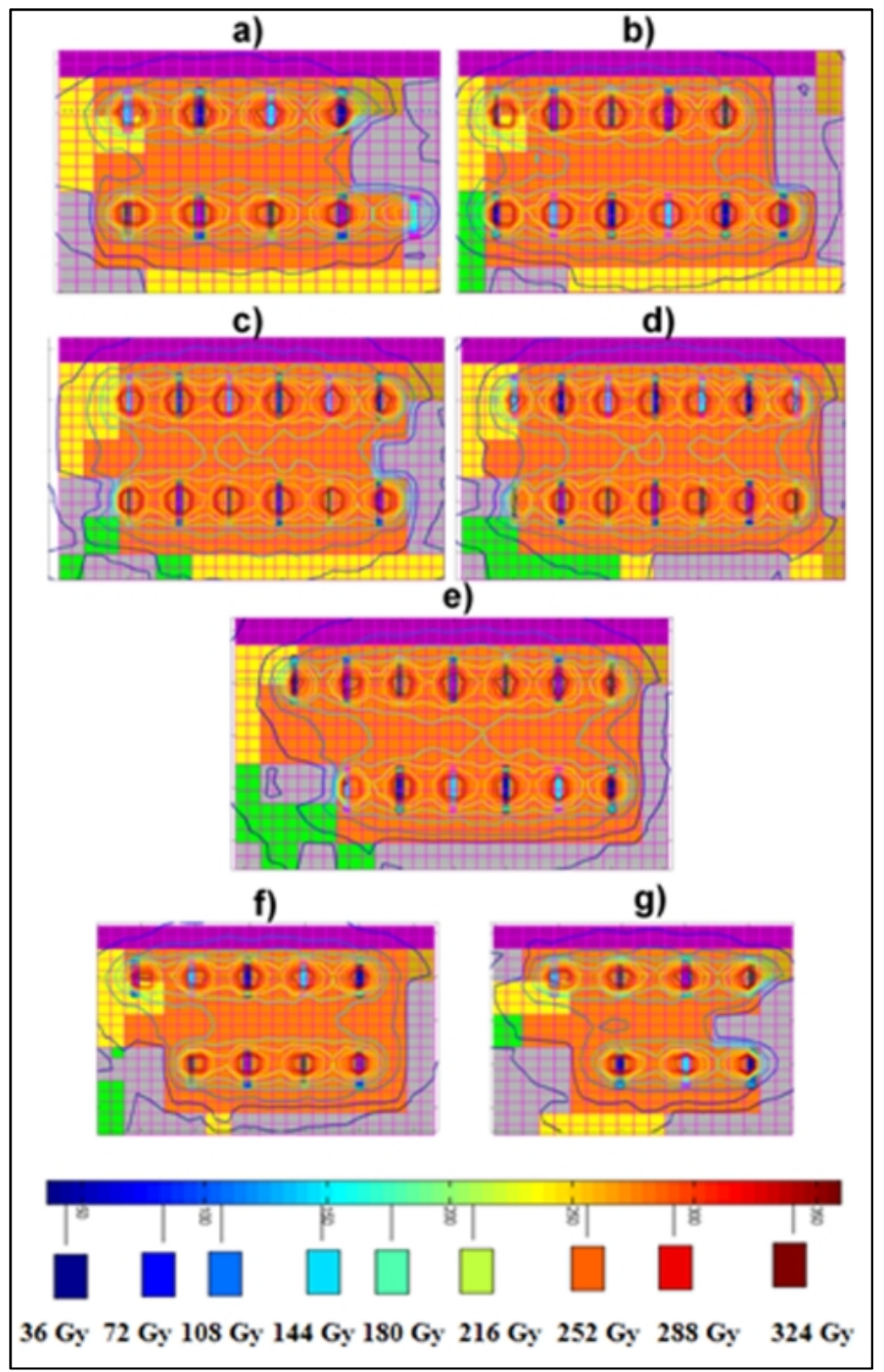

FIG. 10: Isodose curves obtained for each slice of the phantom's prostate region in MAX06 voxels a) $7.0 \mathrm{~cm}$; b) $7.5 \mathrm{~cm}$;) $8.0 \mathrm{~cm}$ d) $8.5 \mathrm{~cm}$; e) $9.0 \mathrm{~cm}$;) $9.5 \mathrm{~cm}$; g) $10.0 \mathrm{~cm}$. 


\section{Conclusions}

With the development of the seed insertion (IS) computational system, it became possible to reduce considerably the replication time for the seeds modeled to perform simulations using the Monte Carlo MCNPX code. In addition, the virtual template developed in the $\mathrm{C}++$ programming language allows replicating any number of sources wished by the user, as well as to reproduce accurately the same spatial distributions performed in real planning.

This computational liberty to analyze and refine parameters, allows quantifying and assessing the distribution of doses obtained between the MCNPX code and the calculation algorithms employed by current planning systems. The modelings' quality assurance in the entry file (input) allows obtaining more realistic results provide by the exit files (output) after performing simulations.

The results obtained with the simulation of the entry file created by the IS system inserted in the phantom MAX06 shows through the acquisition of isodose curves in each slice, which hot spots have values above $300 \mathrm{~Gy}$, as anticipated in literature, stressing the importance of the sources' correct positioning, in which the computational system developed provides, in order not to release excessive doses in adjacent risk organs. The $144 \mathrm{~Gy}$ prescription curve perfectly covers a large percentage of the volume, at the same time that it demonstrates a large decline for short distances, thus allowing the preservation of radio-sensitive organs adjacent to the treatment volume, such a bladder and lymph nodes, reflecting the treatment's efficacy, and hence validating the entry file created by the IS system.

By virtue of this dynamism in the replication process, this system may be added to a planning system likely to be developed in the future, the algorithm of which may be based on the Monte Carlo system for low-dosage rate (LDR) brachytherapy, thus allowing more realistic planning and that takes into consideration the real geometry of sources of radiation.

\section{Conflict of interest}

The authors declare that they have no conflicts of interest. The authors alone are responsible for the content and writing of the paper.

\section{Acknowledgments}

The authors would like to thank the CAPES (Coordenação de Aperfeiçoamento de Pessoal de Nível Superior), CNPQ (Conselho Nacional de Desenvolvimento Científico e Tecnológico) and FAPERJ (Fundação Nacional de Amparo à Pesquisa do Estado do Rio de Janeiro) for their financial support.

\section{References}

1. INCA, Estimativa 2012. Available from http://www.inca.gov.br/estimativa/2012/index.asp?ID=5

2. Pelowitz, DB. Ed. MCNPX ${ }^{\mathrm{TM}}$ User's Manual. Version 2.5.0, Los Alamos National Laboratory Report LA-CP-05-0369 (2005).

3. Stroustrup B. The C++ Programming Language - Third Edition. Addison-Wesley; 1997.

4. Duggan DM. Improved radial dose function estimation using current version MCNP Monte-Carlo simulation: Model 6711 and ISC3500 125I brachytherapy sources. Appl Radiat Isot 2004; 61:1443-50.

5. Reis JP. Avaliações das aproximações utilizadas ao planejamento do tratamento de braquiterapia de próstata. Tese de Doutorado - UFRJ/COPPE/PEN, 2011.

6. Kramer R, Vieira JW, Khoury HJ, Lima FRA, Fuelle D. All about MAX: A male adult voxel phantom for Monte Carlo calculation in radiation protection dosimetry. Phys Med Biol 2008; 48:1239-62.

7. Zubal IG, Harrel CR, Smith EO, Smith AL, Krischlunas P. High resolution, MRI-based, segmented, computerized head phantom, The Zubal Phantom Data, Voxel-Based Anthropomorphic Phantoms. Available from http://noodle.med.yale.edu/phantom

8. Kramer R, Khoury HJ, Vieira J W, Lima VJM. MAX06 and FAX06: Update of two adult human phantoms for radiation protection dosimetry. Phys Med Biol, 2006; 51: 3331-46.

9. Milian FM, Garcia F, Yoriyaz H, Siqueira PTD. Issac S. TOMO_MC programa para criação de INPUT FILES para o MCNPX a partir de modelos natômicos 3D. In: XIII Seminário de Iniciação Científica e 9a Semana de Pesquisa e Pós-Graduação, 2007, Ilhéus. UESC.

10. Dev C++, 2005. GNU - General Public License, Version 4.9, 9.2.

11. Van Riper KA. MORITZ Geometry Tool User's Guide Windows Version (Manual). White Rock Science (2008).

12. Amadei LPP. Evolução Bioquímica através de Medidas Seriadas de Antígeno Prostático Específico (PSA) de Pacientes Submetidos à raquiterapia com o Implante de Sementes de 125 I no ratamento de Adenocarcinoma de Próstata, Tese (Doutorado em Medicina), Faculdade de Medicina da Universidade de São Paulo, Departamento de Radiologia Autarquia Associada à Universidade de São Paulo. (2008).

13. Yu Y, Anderson LL, Li Z, Mellenberg DE, Nath R, Schell MC, Waterman FM, Wu A, Blasko JC. Permanent prostate seed implant brachytherapy: report of the American Association of Physicists in Medicine Task Group No. 64. Med Phys 1999:26: 2054-76. 